gr-qc/9911054

\title{
On Wigner's clock and the detectability of spacetime foam with gravitational-wave interferometers
}

\author{
Y. Jack Ng${ }^{(a),(b) *}$ and H. van $\operatorname{Dam}^{(b)}$ \\ (a) Center for Theoretical Physics, Laboratory for Nuclear Science and Department of Physics, \\ Massachusetts Institute of Technology, Cambridge, MA 02139 \\ (b) Institute of Field Physics, Department of Physics and Astronomy, \\ University of North Carolina, Chapel Hill, NC 27599-3255
}

\begin{abstract}
A recent paper (gr-qc/9909017) criticizes our work on the structure of spacetime foam. Its authors argue that the quantum uncertainty limit for the position of the quantum clock in a gedanken timing experiment, obtained by Wigner and used by us, is based on unrealistic assumptions. Here we point out some flaws in their argument. We also discuss their other comments and some other issues related to our work, including a simple connection to the holographic principle. We see no reason to change our cautious optimism on the detectability of spacetime foam with future refinements of modern gravitational-wave interferometers like LIGO/VIRGO and LISA.
\end{abstract}

Key words: quantum spacetime foam; clocks; gravitational-wave detectors; foundations, theory of measurement, miscellaneous theories

PACS: 42.50.L; 04.80.N; 06.30.F; 03.65.Bz

${ }^{*}$ Corresponding author. E-mail: yjng@physics.unc.edu 


\section{INTRODUCTION}

In their recent paper [1] "On the detectability of quantum spacetime foam with gravitational-wave interferometers," Adler, Nemenman, Overduin, and Santiago claim that the way we use Wigner's quantum clock [2] in a gedanken timing experiment is not justified, thus casting doubt on the detectability of spacetime foam with gravitational-wave interferometers. In particular, they claim that the quantum uncertainty limit for the position of the quantum clock is actually much smaller than that obtained by Wigner and used by us. Since we [3, t] were the first to propose using Wigner's clock to explore the quantum structure of spacetime and to conclude that classical spacetime breaks down into "quantum foam" in a manner quite different from the canonical picture [5], we feel a special obligation to respond to the criticism and to clarify the physics behind our proposal. We will show that the arguments by Adler et al. are invalid.

But first, we should make it clear that we merely want to find out what the low-energy limit of quantum gravity can tell us about the structure of spacetime. For that purpose, it suffices to employ the general principles of quantum mechanics and general relativity. We have little to say about, and for this work, have no use for, the correct theory of quantum gravity (be it string theory, Ashtekar variables/loop-gravity formalism, or something else). We have in mind the low-energy limit of quantum gravity as manifested in the low-frequency spectrum of the displacement noise levels registered in the gravitational-wave interferometers.

In the next section, we recapitulate our previous work [3, [4,6] on spacetime measurements and spacetime foam. In Section III, we respond to each of the four objections against our work raised by Adler et al. In Section IV, we answer some further questions which we think the readers may ask. We offer our conclusions in Section V. We point out that our spacetime foam model [3, [4,6] is consistent with the holographic principle [7]. 


\section{SPACE-TIME MEASUREMENTS AND THE FOAMINESS OF SPACETIME}

Suppose we want to measure the distance between two separated points A and B. To do this, we put a clock (which also serves as a light-emitter and receiver) at A and a mirror at B. A light signal is sent from A to B where it is reflected to return to A. If the clock reads zero when the light signal is emitted and reads $t$ when the signal returns to $\mathrm{A}$, then the distance between $\mathrm{A}$ and $\mathrm{B}$ is given by $l=c t / 2$, where $c$ stands for the speed of light. The next question is: What is the uncertainty (or error) in the distance measurement? Since the clock at $\mathrm{A}$ and the mirror at $\mathrm{B}$ are the agents in measuring the distance, the uncertainty of distance $l$ is given by the uncertainties in their positions. We will concentrate on the clock, expecting that the mirror contributes a comparable amount to the uncertainty in the measurement of $l$. Let us first recall that the clock is not stationary; its spread in speed at time zero is given by the Heisenberg uncertainty principle as

$$
\delta v=\frac{\delta p}{m} \gtrsim \frac{\hbar}{2 m \delta l}
$$

where $m$ is the mass of the clock. This implies an uncertainty in the distance at time $t$,

$$
\delta l(t)=t \delta v \gtrsim\left(\frac{\hbar}{m \delta l(0)}\right)\left(\frac{l}{c}\right),
$$

where we have used $t / 2=l / c$ (and we have dropped an additive term $\delta l(0)$ from the right hand side since its presence complicates the algebra but does not change any of the results). Minimizing $(\delta l(0)+\delta l(t)) / 2$, we get the quantum mechanical uncertainty relation

$$
\delta l^{2} \gtrsim \frac{\hbar l}{m c}
$$

Next, we make use of the principle of equivalence, by exploiting the equality of the inertial mass and the gravitational charge of the clock, to eliminate the dependence on $m$ in the above inequality. This will promote the quantum mechanical uncertainty relation to a quantum gravitational uncertainty relation, making the uncertainty expression useful. Let the clock at A be a light-clock consisting of two parallel mirrors (each of mass $m / 2$ ), a distance of $d$ apart, between which bounces a beam of light. On the one hand, the clock 
must tick off time fast enough such that $d / c \lesssim \delta l / c$, in order that the distance uncertainty is not greater than $\delta l: \delta l \gtrsim d$. On the other hand, $d$ is necessarily larger than the Schwarzschild radius $G m / c^{2}$ of the mirrors ( $G$ is Newton's constant) so that the time registered by the clock can be read off at all: $d \gtrsim \frac{G m}{c^{2}}$. From these two requirements, it follows that

$$
\delta l \gtrsim \frac{G m}{c^{2}}
$$

the product of which and Eq. (3) yields the (low-energy) quantum gravitational uncertainty relation 8

$$
\delta l \gtrsim\left(l l_{P}^{2}\right)^{1 / 3}
$$

where $l_{P}=\left(\frac{\hbar G}{c^{3}}\right)^{1 / 2}$ is the Planck length. The intrinsic uncertainty in space-time measurements just described can be interpreted as inducing an intrinsic uncertainty in the space-time metric $g_{\mu \nu}$. Noting that $\delta l^{2}=l^{2} \delta g$ and using Eq. (5) we get

$$
\delta g_{\mu \nu} \gtrsim\left(l_{P} / l\right)^{2 / 3}
$$

The fact that there is an uncertainty in the space-time metric means that space-time is foamy. Eq. (5) and Eq. (6) constitute our model of spacetime foam. We note that even on the size of the whole observable universe $\left(\sim 10^{10}\right.$ light-years $)$, Eq. (5) yields a fluctuation of only about $10^{-15} \mathrm{~m}$. We further note that, according to our spacetime foam model, spacetime fluctuations lead to decoherence phenomena. The point is that the metric fluctuation $\delta g$ induces a multiplicative phase factor in the wave-function of a particle (of mass $m$ )

$$
\psi \rightarrow e^{i \delta \phi} \psi
$$

given by

$$
\delta \phi=\frac{1}{\hbar} \int m c^{2} \delta g^{00} d t
$$

One consequence of this additonal phase is that a point particle with mass $m>m_{P}\left(m_{P} \equiv\right.$ $\hbar / c l_{P}$ is the Planck mass) is a classical particle (i.e., it suffices to treat it classically). 
Though the fluctuations that space-time undergoes are extremely small, recently Amelino-Camelia has argued (convincingly, we think) that modern gravitational-wave interferometers may soon be sensitive enough to test our model of space-time foam. [9] The idea is fairly simple. Due to the foaminess of space-time, in any distance measurement that involves an amount of time $t$, there is a minute uncertainty $\delta l \sim\left(c t l_{P}^{2}\right)^{1 / 3}$. But measuring minute changes in (the) relative distances (of the test masses or the mirrors) is exactly what a gravitational-wave interferometer is designed to do. Hence, the intrinsic uncertainty in a distance measurement for a time $t$ manifests itself as a displacement noise (in addition to other sources of noises) that infests the interferometers

$$
\sigma \sim\left(c t l_{P}^{2}\right)^{1 / 3}
$$

We can write the displacement noise in terms of its Fourier transform, the associated displacement amplitude spectral density $S(f)$ of frequency $f$. For a frequency-band limited from below by the time of observation $t, \sigma$ is given in terms of $S(f)$ by 10

$$
\sigma^{2}=\int_{1 / t}^{f_{\max }}[S(f)]^{2} d f
$$

For the displacement noise given by Eq. (9), the associated $S(f)$ is

$$
S(f) \sim f^{-5 / 6}\left(c l_{P}^{2}\right)^{1 / 3}
$$

Since we are considering only the low-energy limit of quantum gravity, we expect this formula for $S(f)$ to hold only for frequencies much smaller than the Planck frequency $\left(c / l_{P}\right)$.

We can now use the existing noise-level data [11] obtained at the Caltech 40-meter interferometer to put a bound on $l_{P}$. In particular, by comparing Eq. (11) with the observed noise level of $3 \times 10^{-19} \mathrm{mHz}^{-1 / 2}$ near $450 \mathrm{~Hz}$, which is the lowest noise level reached by the interferometer, we obtain the bound $l_{P} \lesssim 10^{-29} \mathrm{~m}$ which, of course, is consistent with the known value $l_{P} \sim 10^{-35} \mathrm{~m}$. Since $S(f)$ goes like $f^{-5 / 6}$ according to Eq. (11), we can look forward to the LISA generation of gravitational-wave interferometers for improvement by optimizing the performance at low frequencies. (We hope that the gain by going to lower 
frequencies will not be offset by other factors such as a much larger arm length of the interferometers.)

\section{REPLY TO THE COMMENTS BY ADLER ET AL}

In this section we reply to the four points raised by Adler et al. in their paper [可 "On the detectability of quantum spacetime foam with gravitational-wave interferometers."

(1) Ref. [1] claims that if Wigner's clock is quantum mechanical but not free, then the uncertainty limit becomes much smaller than that (Eq. (3)) obtained by Wigner and used by us. In particular, Adler et al. give the example of a quantum clock bound in a harmonic oscillator potential. These authors err in neglecting the fact that the clock is then bound to something. We can now consider that something to be part of the clock (after all, we have already considered the light emission and detection devices as part of our clock), and proceed with our argument presented in Section II.

(2) Ref. [1] claims that Wigner's limit is based on another unrealistic assumption: that the clock does not interact with the environment. In particular, Adler et al. point out that, if the clock is sufficiently large or complex, it will interact with its enviroment in such a way that its wave function decoheres. In addition, these authors claim, such interactions may localize or "collapse" the wave function, resulting in the clock wave function that does not spread linearly over macroscopic times, as opposed to what we have used in Eq. (2). We admit that Adler et al. have raised a good point. But the question of wave function decoherence in the context of fundamental spacetime measurements is quite subtle. We think it is much more reasonable that the phenomenon of enviroment-induced decoherence is an outcome (rather than an input) of quantum spacetime measurements at the fundamental level, with gravity being the universal agent of quantum decoherence as argued in Section II and as emphasized by us [3, [4].

(3) Adler et al. observe that the existing noise-level data [11] obtained at the Caltech 40-meter interferometer can be used to place a lower limit on the effective mass of the 
hypothetical clock in Eq. (3). They find the effective clock mass to be larger than 3 grams which, according to them, is such a remarkably large mass that it hardly seems plausible as a fundamental property of spacetime. This time, these authors err in forgetting that the length scale involved in the Caltech 40-meter interferometer measurement is macroscopic and has nothing to do with fundamental length scales. To appreciate this point, one can use Eq. (3) and Eq. (5) to show that the optimum mass for Wigner's clock (optimum in the sense that it yields the smallest uncertainty in distance measurements) is given by

$$
m \sim m_{P}\left(l / l_{P}\right)^{1 / 3}
$$

Thus the optimum mass of the quantum clock depends on $l$, the distance in the distance measurement. If $l$ is macroscopic, the optimum mass is much larger than the Planck mass. On the other hand, if we dare (recall that we expect our result to be valid only for the lowenergy domain of quantum gravity) to use Eq. (12) in the measurement of a microscopic distance approaching the fundamental length scale $l_{P}$, the optimum mass of the hypothetical clock would approach $m_{P}$, the fundamental mass scale. Therefore, the relatively large mass of the clock found in Ref. [1] is to be expected since the distance involved in the Caltech interferometer measurement at $450 \mathrm{~Hz}$ is huge compared to the Planck length.

The above three objections raised by Adler et al. are all directed at the quantum uncertainty limit (Eq. (3)) obtained by Wigner and used by us. There is a way, albeit an indirect one, to show that the uncertainty limit (Eq. (3)) actually should be quite palatable even to those who believe that the intrinsic uncertainty in distance measurements is independent of the distance being measured and is given simply by the Planck length. All it takes is to use Eq. (3) as the starting point. But for the bound on $m$, instead of Eq. (田), one uses

$$
l \gtrsim \frac{G m}{c^{2}}
$$

which is nothing but the mathematical statement of the obvious observation that, to measure the distance from A to B, point B should not be inside the Schwarzschild radius of the clock at A. Then one finds 


$$
\delta l \gtrsim l_{P}
$$

the canonical uncertainty [5] in distance measurements. Thus the only question remaining is whether the more restrictive bound on $m$ given by Eq. (4) is also correct. This brings us to the next comment.

(4) Adler et al. note that the presence of the measurement clock system certainly produces a distortion of spacetime, but Eq. (4) tells us that it also produces an uncertainty in spacetime distances of about the same amount. (This fact has not escaped our attention. See Ref. [3] and [4.) They contend that Eq. (4) must be wrong. In particular, taking the spinning Earth as the quantum clock, they assert that, with Eq. (丰), one would conclude that objects in the vicinity of the Earth have a minimum intrinsic position uncertainty of roughly the Schwarzschild radius of the Earth, which is about $1 \mathrm{~cm}$, and this is manifestly false by many orders of magnitude. Our reply is simply that one cannot use the spinning Earth, excellent as it is as a clock for daily lives, as Wigner's quantum clock in the gedanken timing experiment. For one thing, the spinning Earth, by itself, cannot function as a clock. One also needs the Sun or the stars, for example, thus complicating the already huge timing device. As a clock, the spinning Earth is not very accurate. Let us imagine building a telescope with opening as large as the earth. For visible light, its resolving power is about $10^{-14}$ radians. The Earth and the telescope rotate by about $10^{-4}$ radians per sec. Hence, the spinning Earth, as a clock, cannot be precise beyond the $10^{-10}$ sec. level, which can be translated to yield a distance measurement accuracy to about $1 \mathrm{~cm}$, hardly the precision needed for spacetime measurements at the fundamental level. Intuitively, it is also clear that a quantum spacetime measurement cannot tolerate the use of a monstrously huge and massive clock like the spinning Earth which causes such a distortion in the geometry of spacetime that it completely overwhelms the uncertainty in distance measurements. Recall that even on the size of the observable universe, the end result of our analysis yields a distance fluctuation of only about $10^{-15} \mathrm{~m}$ which is much smaller than the Schwarzschild radius of the Earth. It is true that we have merely used the light-clock as a model clock and 
there may be more ideal clocks to use; but due to its simplicity, the light-clock fits the bill of a quantum clock for the gedanken timing experiment at the fundamental level.

\section{COMMENTS ON SOME OTHER QUESTIONS}

In this section, we comment on four more questions that we think some of our readers may ask.

(1) In Section II, we require our light-clock to tick off time fast enough such that $d / c \lesssim \delta l / c$, implying that $d / c$ is the smallest unit of time for our light-clock. Some readers may well ask whether it is not possible to have smaller units of time by taking fractions of $d / c$. If it is possible, then the inequality $d / c \lesssim \delta l / c$ needs not hold. Our reply is that, to be accurate, $d / c$ is indeed the smallest unit of time for our light-clock. To make an analogy, one does not use a minute-clock to time a 100-m dash which takes only about $10 \mathrm{sec}$, a fraction of a minute.

(2) Recall that our use of the light-clock in the gedanken timing experiment yields Eq. (四). One may wonder if the ensuing result (Eq. (5)) is not just an artifact of our model clock. Thus it is logical to ask whether it is not possible to replace our light-clock with some other types of clocks such that all those inequalities (including the Schwarzschild bound) no longer hold. In the absence of explicit examples, it is hard to draw any conclusions. But let us consider a clock made of a small object revolving around a black-hole just outside its event-horizon. (And let us ignore the gravitational radiation problem.) Timing is provided by the periodicity of the motion. Then there is no analog of $d$, the separation of mirrors in the light-clock, and it follows that those inequalities are no longer valid, so goes the

hypothetical argument. The trouble with this argument is that actually there is an analog of $d$, given by the size of the orbit around the black-hole; and mass of the clock here is that of the black-hole. Therefore it follows that those inequalities in Eqs. (四) and (5) (as an order of magnitude estimate) still hold.

(3) In Eq. (10), we have used $1 / t$ as the lower limit of integration; but what if the lower 
limit is actually a multiple (call it $n$ ) of $1 / t$ ? The answer is that, since Eq. (9) holds only up to a multiplicative factor of order 1 , a short calculation shows that so long as the multiple $\mathrm{n}$ is no more than 2 orders off unity, Eq. (10) stands as it is.

(4) One may worry that the metric fluctuations given by Eq. (6) yield an unacceptably large fluctuation in energy density. Since we asked ourselves this very question and answered it in Ref. [4] already, we will be very brief here. But let us generalize the discussion to metric fluctuations of the form parametrized by $a$ with $0<a \leq 1$

$$
\delta g \gtrsim\left(l_{P} / l\right)^{a}
$$

(corresponding to distance uncertainties of $\delta l \gtrsim l^{1-a} l_{P}^{a}$ and displacement amplitude spectral densities of $\left.S(f) \sim c^{1-a} l_{P}^{a} f^{a-3 / 2}\right)$. Models with larger spacetime fluctuations are parametrized by smaller values of $a$. We note that, for our model of spacetime foam, $a=2 / 3$, while, for the canonical model, $a=1$. The case $a=1 / 2$ corresponds to the model of spacetime foam considered in Ref. [12]. Regarding the metric fluctuation as a gravitational wave quantized in a spatial box of volume $V$, one finds [4] that the energy density is given by

$$
\rho \sim\left(\frac{m_{P} c^{2}}{V}\right)
$$

for $1 / 2<a \leq 1$. Thus the energy density associated with metric fluctuations given by Eq. (15) is obviously small in the large volume $\left(V>>l_{P}^{3}\right)$ limit which we have assumed. Note that the energy density is of the form given by Eq. (16) and holds, as an order of magnitude estimate (consistent with what we have been using), independent of the parameter $a$ so long as $a$ is not too close to $1 / 2$. For $a=1 / 2$, one gets

$$
\rho \sim\left(\frac{m_{P} c^{2}}{V}\right) \ln \left(\frac{V^{1 / 3}}{l_{P}}\right) .
$$

For $0<a<1 / 2$, one finds

$$
\rho \sim\left(\frac{m_{P} c^{2}}{V}\right)\left(\frac{V^{1 / 3}}{l_{P}}\right)^{1-2 a} .
$$

The trend is clear: in general, larger spacetime fluctuations cost more energy. Note that the energy density $\rho$ associated with metric fluctuations (Eq. (15)) is the smallest for the range of $a$ which includes the canonical model and our model of spacetime foam. 


\section{CONCLUSIONS}

In Ref. [四], Adler et al. raise four objections to our work on spacetime measurements and spacetime foam. Three of the objections are related to the question whether the quantum uncertainty limit obtained by Wigner and used by us is valid. These authors also criticize the gravitational uncertainty limit (Eq. (丑) obtained by us; they conclude that it is an artifact of our choice of a particular type of hypothetical clock and is, therefore, non-fundamental in nature. While they have raised some good points, we believe their argument is flawed (as shown in Section III). We agree that the question of an ideal quantum clock is not yet settled. But it is just inappropriate to use the spinning Earth as a quantum clock in a fundamental spacetime measurement. History has taught us that fundamental physics is best explored with simple devices; our light-clock is a simple device.

Since all the criticism by Adler et al. is related to issues of clocks, perhaps a better argument for a spacetime foam different from the canonical model is one that does not use clocks. As shown in Section IV, the energy density associated with spacetime quantum fluctuations takes on the smallest (and comparable) values for those spacetime foam models with the parameter $a$ in the range $1 / 2<a \leq 1$ so long as $a$ is not too close to $1 / 2$. So, it is possible that Nature chooses to have a larger spacetime fluctuation (than that predicted by the canonical model) at a comparable cost of energy. This argument is very loose, but hopefully we have made our point. Only future experiments can tell which value of $a$ (i.e., which spacetime foam model) Nature picks. At present, if we assume that the distance uncertainty expressions given above are not off by more than an order of magnitude, a short calculation shows that the existing data provided by the Caltech 40-meter interferometer rule out models with $a<0.54$. We can expect more stringent bounds on $a$ with modern gravitational-wave interferometers.

There is one theoretical consideration which sets our model of spacetime foam $(a=2 / 3)$

apart from the others. It is its connection to the holographic principle [7] which asserts that the number of degrees of freedom of a region of space is bounded (not by the volume 
but) by the area of the region in Planck units. To see that, let us consider a region of space with linear dimension $l$. According to the conventional wisdom, the region can be partitioned into cubes as small as $l_{P}^{3}$. It follows that the number of degrees of freedom of the region is bounded by $\left(l / l_{P}\right)^{3}$, i.e., the volume of the region in Planck units. But according to our spacetime foam model [3:4:6], the smallest cubes inside that region have a linear dimension of order $\left(l l_{P}^{2}\right)^{1 / 3}$. Accordingly, the number of degrees of freedom of the region is bounded by $\left[l /\left(l l_{P}^{2}\right)^{1 / 3}\right]^{3}$, i.e., the area of the region in Planck units, as stipulated by the holographic principle. Thus one may even say that the holographic principle has its origin in the quantum fluctuations of spacetime. Evidence for our spacetime foam model would lend experimental support for the holographic principle.

Finally we recall that spacetime (metric) fluctuations can be regarded as a kind of quantized gravitational waves. It is uncanny that, through future refinements, modern gravitational-wave interferometers like LIGO, VIRGO, and LISA, which are designed to detect gravitational waves from neutron stars, supernovae, black-holes, and the like, may also be able to detect, as a by-product, a very different kind of gravitational waves - the kind that encodes the quantum fluctuations of spacetime.

\section{Acknowledgments}

One of us (YJN) thanks R. Weiss for a useful discussion. This work was supported in part by the U.S. Department of Energy under \#DF-FC02-94ER40818 and \#DE-FG0585ER-40219, and by the Bahnson Fund of the University of North Carolina at Chapel Hill. Part of the work was carried out by YJN while he was on leave of absence at MIT. He thanks the faculty at the Center for Theoretical Physics for their hospitality. 


\section{REFERENCES}

[1] R. J. Adler, I. M. Nemenman, J. M. Overduin, and D. I. Santiago, gr-qc/9909017.

[2] E. P. Wigner, Rev. Mod. Phys. 29 (1957) 255; H. Salecker and E. P. Wigner, Phys. Rev. 109 (1958) 571.

[3] Y. J. Ng and H. van Dam, Mod. Phys. Lett. A9 (1994) 335; in Proc. of the Fundamental Problems in Quantum Theory, eds. D. M. Greenberger and A. Zeilinger, Ann. New York Acad. Sci. 755 (1995) 579.

[4] Y. J. Ng and H. van Dam, Mod. Phys. Lett. A10 (1995) 2801; Europhys. Lett. 38 (1997) 401.

[5] C. W. Misner, K. S. Thorne, and J. A. Wheeler, Gravitation (W. H. Freeman, 1973), pp. 1190-1194.

[6] Y. J. Ng and H. van Dam, gr-qc/9906003, to appear in Found. Phys.

[7] G. 't Hooft, in Salamfest 1993, p.284, gr-qc/9310026; L. Susskind, J. Math. Phys. 36 (1995) 6377.

[8] A very different way to obtain this result can be found in F. Karolyhazy, IL Nuovo Cimento A42 (1966) 390. See also N. Sasakura, Prog. Theor. Phys. 102 (1999) 169, and Preprint hep-th/0001161.

[9] G. Amelino-Camelia, Nature 398 (1999) 216.

[10] V. Radeka, Ann. Rev. Nucl. Part. Sci. 38 (1988) 217.

[11] A. Abramovici, et. al., Phys. Lett. A218 (1996) 157.

[12] G. Amelino-Camelia, Mod. Phys. Lett. A9 (1994) 3415. Also see L. Diosi and B. Lukacs, Phys. Lett. A142 (1989) 331. 